\title{
Hacia la edificación institucional del Sistema de Pagos en México I 905-1917
}

\author{
Did the instotucional building of the Payment System in Mexico I905-1917
}

Jimmy Melo*

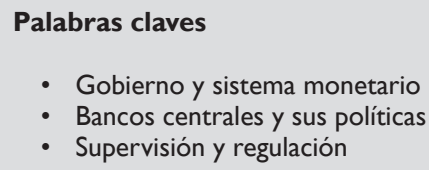

\begin{abstract}
Resumen
Este artículo explora cómo el Sistema de pagos se fue configurado en México de 1905 a 1917. Mediante la idea de que la moneda es un medio de cancelación final de pagos, se evalúa el papel de los acuerdos institucionales para traer estabilidad y la relación entre esos acuerdos con la liquidez inyectada al sistema. Se presenta también un enfoque parcial del sistema mexicano de pagos que se enfoca en el sistema bancario, y cómo esto define el espacio de las estrategias en el periodo posrevolucionario.
\end{abstract}

Abstract

This paper explores how the system of pays was configured in México from 1905 to 1917. Using the notion that money is a medium of final cancelation of pays, it evaluates the role of institutional agreements to bring stability to the system, and the relation between these agreements and liquidity injected to the system. It presents a partial drawn of Mexican system of pays focusing on the bank system and how it defined the space of strategies in the postrevolutionary period.

Key words

- Goverment and monetary system

- Central Banks and their policies

- Goverment policy and regulation

JEL: E42, E58, G28

\section{Introducción}

Este trabajo intenta responder si en el tránsito del Porfiriato a los primeros gobiernos revolucionarios se logra establecer un arreglo institucional en cuanto a una unidad de cuenta como forma monetaria capaz de desempeñar funciones de medio de pago y reserva de valor. "El desocupado lector" que siga las líneas de este escrito encontrará una respuesta afirmativa con algunos matices, que bien pueden ser leídos como una respuesta negativa, dado que el cambio institucional se evidencia como un proceso lento, gradual y lleno de avatares, en el cual los arreglos institucionales no necesariamente son estables a largo plazo o promotores del desarrollo económico.

El punto central de este texto, reside en el hecho de que las soluciones a ciertos problemas de orden económico responden a elementos coyunturales y estructurales sobre los cuales se erigen arreglos institucionales que definen el espacio de estrategias de las partes en negociación. En ese sentido, si la economía de la época avanza en la incorporación de elementos de corte capitalista cabe preguntarse si el sistema de pagos se articula con este proceso y de qué manera lo hace. En particular, la definición de una forma monetaria pasa por su capacidad de especificar derechos de propiedad, es decir, el uso extensivo de una unidad de cuenta que permita definir acuerdos de pago.

Así, el primer elemento del análisis es el intento de consolidación del patrón oro-plata en mexicano a través de la ley monetaria de 1905, en esta ley se especi- 
fican las unidades de medida y las equivalencias entre formas monetarias. Además, establece el alcance liberatorio de cada una de ellas, incluyendo los billetes de banco, lo cual es evidencia de las necesidades de liquidez en la economía mexicana de la época. En esa dirección se evalúa el rol del sistema bancario. Con ello, se establece un bosquejo del sistema de pagos. Este bosquejo es incompleto y queda abierto a futura investigación el rol de otros agentes que no están claramente identificados o incorporados por el sistema legal como lo son los mercados informales de crédito y las casas cambiarias.

El segundo elemento es la evaluación de la estabilidad de los acuerdos entre las diferentes entidades que conforman el sistema de pagos, en particular, como ciertos acuerdos sobreviven al proceso revolucionario. Como hipótesis adelantamos que el sistema bancario fue capaz de incorporar las necesidades de liquidez de las diferentes facciones en punta para diseñar las estrategias contingentes que le permitieron validar acuerdos heredados del periodo porfiriano.

Así pues, en la sección 2 se analiza el fenómeno monetario en el Porfiriato, delimitando las instituciones de interés y el espacio de estrategias en este lapso temporal; la sección 3 proyecta estas instituciones hacia el periodo revolucionario, evaluando su estabilidad; con ello la sección 4 presenta algunas conclusiones a la luz de los elementos estratégicos de la propuesta posrevolucionaria de Sistema de Pagos.

\section{Consolidación de la moneda en el Porfiriato: | 905-| 9 | 2}

Aunque en este periodo se dio la extensión de derechos de propiedad privada en la escala urbana y algunos núcleos locales de producción agropecuaria vinculados con producción para consumo doméstico y de exportación, un porcentaje importante de la producción rural se encontraba en aislamiento de esta dinámica toda vez que subsistían modelos de sujeción de mano de obra y un profundo estancamiento del mercado de tierras. Se evidencia relativo avance en la consolidación de los preceptos liberales de la Constitución de 1857 y el afianzamiento de características de corte capitalista en la economía mexicana a finales del siglo XIX y principios del $x x$. Paulatinamente, México transforma sus características de economía de antiguo régimen, transformación que aumenta los requerimientos de financiación. En este escenario, el dinero de cuenta adquirió una mayor preponderancia, ya que a través de éste se especifican y estandarizan los derechos de propiedad y se cubre la distancia entre los gastos de inversión y el retorno de las utilidades. Este tránsito a una economía cuyo rasgo fundamental es la incertidumbre,' obedecía a tres factores: (i)

I Como indica Fontana "... once the choice is made there is no guarantee that plans and expectations will be realized. The realization process is constantly disrupted and remains characterized by fluidity 
Crecimiento económico sostenido, el cual fue desencadenado y acompañado, por (ii) una mayor integración del mercado interno y (iii) una mayor integración de la economía mexicana al mercado mundial.

Bajo estas consideraciones, el Sistema de Pagos Porfiriano debió satisfacer las necesidades de liquidez tanto del gobierno como de los inversores privados. La articulación del mercado interno exigió el desarrollo de mecanismos de financiación y un medio de pago válido a lo largo y ancho del país. La mayor integración a los mercados internacionales reclamó la estandarización de la unidad de cuenta con los sistemas de pago internacionales; en otros términos, se reclamó la estabilidad del tipo de cambio.Adicionalmente, la expansión ferrocarrilera requería mecanismos de financiación adecuados al manejo de los recursos externos que la financiaron, así como para los nuevos proyectos productivos que se yuxtaponían a éste.

En el campo institucional, la Constitución de 1857, en lo que compete al Sistema de Pagos, apenas si reconoce la necesidad de un monopolio en la acuñación de la moneda (Articulo 28), faculta al congreso "para establecer casas de moneda, fijar las condiciones que esta deba tener, determinar el valor de la extranjera, y adoptar un sistema general de pesos y medidas" (Artículo 72, Numeral XXIII) al igual que establece que los estados no pueden "Acuñar moneda, emitir papel moneda, ni papel sellado" (Artículo II I, Numeral III). Esto muestra un vacío con relación a otras formas de pago que para la época se usaban en otros países; vacío que empieza a llenarse con el Código de Comercio de 1884, la Ley General de Establecimientos de Crédito de 1897 y la Ley Monetaria de 1905, ${ }^{2}$ cuya instrumentación discutiremos más adelante.

Lo dicho hasta aquí, constituye los antecedentes y la base del Sistema de Pagos Porfiriano, el cual vendrá a consolidarse al final de esta época. Antes de describir el Sistema de Pagos, es menester señalar algunas características generales de una economía monetaria, para perfilar el contraste con la economía de la época.Así, en primer lugar, en una economía monetaria el dinero es una promesa de pago entre dos agentes mediada por un tercero. ${ }^{3}$ En segundo lugar, la demanda por liquidez tiene dos

and volatility. Then, if choice is non-empty, agents may transform plans and objects as a result of (historical) time.Agents can accomplish the necessary transactions that are forced on them as they adjust to the unforeseen and unforeseeable." (Fontana 724) Por esta razón en el capitalismo, la fuente del dinero es la producción bajo incertidumbre; a diferencia de las economías tribales, el capitalismo no es posible sin dinero (Wray 1993). Dado que las decisiones tomadas hoy buscan cubrir el lapso entre el momento de los gastos y la percepción de los ingresos, lo cual necesariamente implica asumir riesgos que contribuyen a formar el resultado final determinado por el conjunto de interacciones dentro del sistema económico considerado como un todo.

2 Ley que establece el régimen monetario en los Estados Unidos Mexicanos, 25 de marzo de 1905. La legislación mexicana de Manuel Dublán y José María Lozano.

3 Así, los agentes no tienen la capacidad girar promesas de pago como deuda contra si mismos; si 
componentes “ ( $i$ ) la demanda activa, que depende del nivel de actividad real y planeada, y (ii) la demanda inactiva, que depende del estado de confianza de los poseedores pasivos de títulos y activos" (Keynes: 1937, 24). El componente activo sienta las bases del proceso de creación de la liquidez requerida por los empresarios y el gobierno. Finalmente, "la oferta (de liquidez) depende de las condiciones que los bancos están dispuestos a aceptar para mantener una mayor o menor liquidez" (Keynes: 1937, 24). Bajo estas tres condiciones, la liquidez que se inyecta en el sistema, depende de las negociaciones entre tres macrogrupos: los empresarios, el gobierno y la banca; ${ }^{4}$ mientras que el flujo en el sistema dependerá del componente inactivo, es decir, de los agentes que disponen de su riqueza en forma de depósitos.

Con base en lo anterior, puede afirmarse que en el Porfiriato se edifica un Sistema de pagos heterogéneo cuyos elementos característicos son: el uso de la plata y el oro (en una menor medida) como medios de pago, ${ }^{5}$ el trueque directo en zonas aisladas, algunos activos financieros, ${ }^{6}$ y los billetes. Estos últimos, en sentido estricto, no constituían un medio de cancelación de las deudas, toda vez que los "billetes... circularían en el comercio libremente, hasta que alguno de los tenedores de un billete quería ir a cambiarlo por dinero al banco", 7 es decir, oro o plata.

La extrapolación de estos elementos con relación al lugar que ocupan los agentes en el sistema, permite delinear la arquitectura del Sistema de Pagos Porfiriano, estableciendo varias relaciones de interés tanto a nivel de la teoría monetaria como a nivel histórico.Así, se clasifican las principales formas monetarias de acuerdo a su grado de liquidez, es decir, el grado en que estos activos pueden transformarse en otros bienes. El grado de liquidez en este caso depende de las condiciones de circulación que se imponen a cada forma monetaria. La introducción de esta variable es de interés en cuanto permite fijar una escala de orden de la prima de liquidez entendida como "The amount... which they are willing to pay for the potential convenience or security given by this power of disposal (...), we shall call its liquidity-premium l." (Keynes

cada empresa emite su propio titulo de deuda para realizar una transacción, este título funciona como medio de cambio pero no como medio para la cancelación final del pago asociado a dicha transacción, el símbolo (titulo) emitido por la empresa no debe brindarle privilegios de acuñación (Graziani, 1990) 4 "La banca aparece aquí como una institución social que regula el intercambio (y la financiación) a través de la creación y destrucción de los medios de pago necesarios” (Lorente: | 99|)

5 Los cuales, dado su valor intrínseco, constituyeron medios de cancelación final de las deudas.

6 Muchos de los cuales tenían liquidez limitada y de corto plazo; en este periodo las empresas empiezan a cotizar en las bolsas extranjeras y las operaciones de redescuento de estos papeles eran una actividad de importancia para la banca.

7 Toribio Esquivel Obregón, Tomado de Anaya (2002, 9). Como indica Rachline “Qu'est-ce qu'un billet de banque à l'origine? Une promesse de payer, un engagement que prend la banque de restituer le dépôt sur présentation du billet. Le métal est la <<vraie>> monnaie; le billet n'est qu'une prommesse de métal" (Rachline 1982, 460) 
1936 Cap. 17). Veamos como se establece la prima de liquidez, a qué tipo de arreglo institucional obedece y cómo afecta la relación entre los agentes.

De acuerdo a la Ley Monetaria de 1905 “Las monedas de oro de cualquier valor y las de plata tienen poder liberatorio ilimitado... En cuanto las otras monedas de plata, á las de níquel y á las de bronce, sólo es obligatorio su admisión en un mismo pago en cantidad no mayor de veinte pesos para las monedas de plata ni de un peso para las de níquel y las de bronce" (Art. 20, Ley 1905). Con ello, la ley determina que el dinero de alto poder es el oro a nivel legal y reconoce el papel de la plata como medio aceptado en el país, mientras que otras monedas tendrían un papel secundario, limitado a intercambios de pequeña escala. Sin embargo, es de destacar que la prima de liquidez del oro, en buena parte del periodo será inferior a la de la plata. El hecho de que México fuera uno de los principales productores de plata da un matiz que debe trabajarse con cuidado, dado que el dinero mercancía presupone que cualquier agente puede producir el bien que funciona como dinero y bastaría con incrementar la cantidad de trabajo aplicado en la producción de dicha mercancía para obtener mayor liquidez. Naturalmente la plata constituye una doble fuente de recursos como "mercancía" exportada y como medio de pago en el mercado mundial y nacional, de allí que los precios internacionales de la plata generen una presión constante sobre el sistema de pagos mexicano; si bien podía producirse más plata, la liquidez generada fluía hacia los mercados internacionales, particularmente durante las coyunturas de precios "favorables" la moneda acuñada era drenada del sistema. De igual forma, en este marco histórico el desarrollo de los billetes como forma monetaria se acompañó y limitó por el uso extendido de la plata como medio de pago.

Al hablar de la plata y el oro como medio pago debemos considerar el de las casas de moneda. En este caso, el monopolio de acuñación pertenece al Ejecutivo de la Unión y se reglamenta en el artículo 9 de la Ley monetaria de $1905,{ }^{8}$ mientras que según el artículo 19 de la misma ley, las casas de moneda continúan prestado servicios de fundición, ensaye y afinación. Kemmerer (1917) señala que las casas de moneda acuñaban monedas cuando los particulares presentaban la plata o el oro que requerían, lo cual permitía que la inyección de liquidez al sistema respondiera a las necesidades de la actividad económica. Además, se prevé la creación del fondo regulador de la circulación monetaria, "cuyo objeto fundamental es facilitar la adaptación de la circulación monetaria, en cuanto á la cantidad de moneda, á las exigencias de la estabilidad del tipo cambio exterior" (Art. 27, ley 1905). A este respecto, una organización de importancia en este periodo fue la Comisión de Cambios y Moneda, a la

8 En virtud de los artículos 28 y 72 de la constitución de 1857.

9 La Comisión se encargaba de la compra de metales preciosos; fijaba la cantidad de acuñación para la circulación; recogía las monedas desgastadas, cambiaba la moneda fuerte por fraccionaria y adminis- 
cual se le confirió la administración de dicho fondo y así, la regulación de la circulación monetaria (Marichal, 2004).

En el caso de los billetes de la banca de emisión debemos evaluar la situación con mayor detenimiento. En primer lugar la ley establecía "El billete de banco es de circulación enteramente voluntaria y, por tanto, en ningún caso se considerará como forzosa su admisión por el público" (Artículo 19, Ley 1897) De allí que la prima de liquidez de los billetes de banco sea menor a la del oro o la plata, es decir, es una función creciente de la confianza que el público deposita en los bancos de emisión y decreciente en la prima de liquidez del oro y la plata. En segundo lugar, debemos considerar la posición de cada banco en el mercado, en particular, evaluar la posición de los Bancos Nacional de México (Banamex) y Londres y México, dado que éstos operaban bajo concesiones especiales de emisión de billetes de circulación nacional, elemento definitorio de la prima de liquidez de los billetes emitidos por estos bancos. Adicionalmente, los bancos de emisión estatal estaban restringidos a las acciones de estos dos bancos, en particular, a Banamex, en la medida que al cambiar sus billetes los bancos estatales debían incurrir en elevados costos de transacción para cubrir sus saldos a favor de los dos Bancos; hecho que fuera evidenciado en 1908 por Limantour:"la acumulación de billetes de los distintos bancos en poder de otras instituciones del mismo género, práctica que aumenta ficticiamente la circulación y que ofrece peligro de que los bancos puedan hostilizarse recíprocamente" (Limantour, 1908) 10

Esta situación motivó la creación del Banco Central en 1899, con el fin de que éste permitiera resolver los problemas de liquidez de la banca estatal, ya que éste aceptaría los billetes de los bancos estatales, y actuaría como agente comercial de éstos en la capital donde obtenían los pesos fuertes (Anaya, 2002, 43). Ante las presiones de los Bancos Banamex y Londres y México, la solución estratégica para los bancos estatales consistió en una organización que les permitió menguar los costos de transacción asociados al mercado oligopólico. Con la creación del Central se evidenció un cambio en el comportamiento de la banca estatal, ésta menos aversión al riesgo, emitía en el límite de lo establecido por la ley (Maurer, 2002, 6I-66). No obstante, fueron muchos los problemas por los que el Banco Central atravesó, entre otros la falta de capital y la carencia de mecanismos de coordinación con la banca estatal, lo que llevaría a su liquidación en 1913."

traba el Fondo Regulador.

I0 Para evitar dicha práctica, continua Limantour, "se previene el canje periódico de los billetes y al pago de todos los saldos, todo mediante un sistema de liquidación que el ejecutivo fijará reglamentariamente" Aunque en la práctica nunca se reglamentó el susodicho canje y la periodización de éste aumentó los costos de transición de la banca de emisión estatal.

II "The bank operated with extremely low specie reserve ratios, (...), and invested large amounts in 
Para evaluar el poder de mercado de Banamex se cuenta con las Tobin's $q$ estimadas por Maurer, la cual es el cociente entre el valor de mercado de la empresa y los costos de reemplazamiento de sus activos. Así, si en una actividad donde la $q$ es mayor a la unidad existen incentivos a la entrada y en un mercado sin barreras a la entrada o la salida ésta será igual a la unidad. El análisis de media de las q’s en el periodo de 1902-1910, ${ }^{12}$ permite evaluar la estructura de mercado de la banca Porfiriana. Como se reseña en la gráfica I, la $q$ promedio de Banamex $q_{B X}=1.3$ en este periodo estuvo por encima del promedio de los demás bancos de la muestra, mostrando el liderato de Banamex y las barreras a la entrada en el mercado nacional establecidas por los acuerdos entre la Banamex y el gobierno.

\section{Gráfica I}

Análisis de Media Tobin’s q banca: 1902-1910

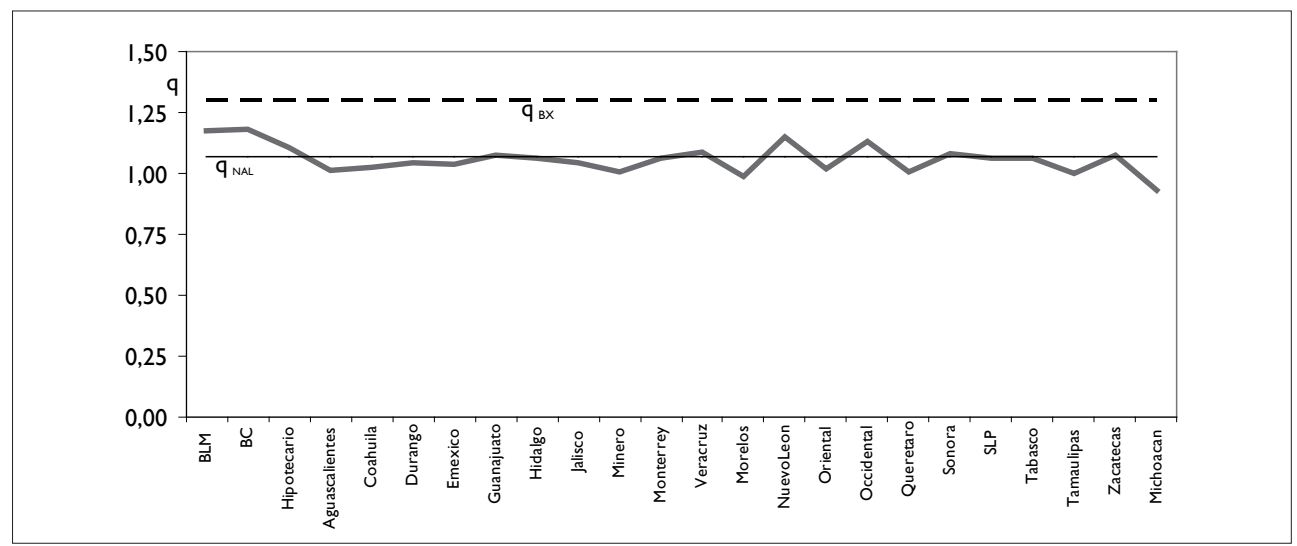

Fuente: Maurer (2002). Cálculos del autor.

illiquid long-term agricultural and industrial ventures. When a crisis finally came, in 1907, the Banco Central proved unable to provide the estate banks with the support thy needed. The Banco Central was a central bank only in name, no in function, handicapped by its relatively small size, inability to impose required reserve ratios, and inability to issue paper currency" (Maurer 2002, 66) Es por esta razón que aún en 1908 Limantour percibe los problemas de la banca estatal en cuando al canje de sus billetes.

12 Ajustando modelos de diferencia en las medias, para una muestra de 25 bancos en el periodo de 1902-1910, que permitan evaluar la estructura del mercado. El primer modelo $q_{i, t}=q_{B X}+\sum_{i i_{i} B_{i}}^{24}$ $B_{1}=1_{\text {banco=banco }}$, donde, el cual puede reescribirse como el sistema $\bar{q}_{i}=\bar{q}_{B X}+\mathrm{a}_{i}$, todos los alfas son negativos y estadísticamente significativos (ver anexo I Análisis de media I). El segundo modelo toma

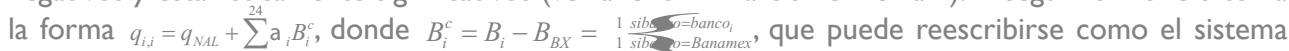
$q_{i}=q_{N A L}+\mathrm{a}_{i}$, a diferéncia del primero evalúa la relación de la media de cada banco con la media nacional (media centrada) y contra la de Banamex, ésta aparece como una variable de instrumentación (ver anexo I Análisis de media 2). 
La media nacional se ubica en I.06; muy por debajo de Banamex. En el análisis de media centrada, la mayoría de los bancos presenta diferencias con respecto a la media nacional no significativas ( 14 bancos de 24) y unos pocos con diferencias negativas y significativas (7 de 24), aunque siguen en el rango de $q$ mayor a la unidad. Con ello, puede concluirse que la mayoría de los bancos estatales presenta algún grado de poder de mercado, resultante de la existencia de barreras a la entrada a lo largo del sistema bancario nacional y estatal. ${ }^{13}$ Contrastante a la situación de la banca estatal es la situación del Banco de Londres y México (BLM) y el Banco Central (BC), los cuales muestran diferencias positivas $(0.1$ y 0.12$)$ y significativas con respecto a la media nacional. Así, para evaluar este resultado, contrastamos la media de Banamex con las medias de los Bancos de Londres y México, el Central y los demás bancos considerados en un solo grupo, estimando el modelo:

(I) ${ }^{.14} q_{i, t}=1.3-0.12 B L M-0.11 B C-0.25 B E$

Es claro que los privilegios de Banamex y el BLM los colocan en la cima del sistema bancario, mientras que el $\mathrm{BC}$ aparece como una estrategia de la banca estatal que fue en cierto grado efectiva para competir y menguar los efectos de éste oligopolio, construido sobre la base de privilegios transados en el mercado político. Como reseña Maurer "In short, the privileges granted Banamex were the product of a political process (...) Scientific laws and abstract debates did not determine the shape of Porfirian banking. Politics did." (Maurer, 2002, 49).

Evaluemos ahora la situación de confianza en el sistema bancario, en este caso nos remitimos a la noción de prima liquidez, toda vez que esta mide el pago que se está dispuesto a hacer por la seguridad que brinda poseer determinado activo. El estado de confianza necesariamente es el reflejo del oligopolio a la cabeza de Banamex. Con los argumentos hasta aquí reunidos podemos plantear la hipótesis de que ésta situación implicaba una prima de iliquidez muy elevada para las notas bancarias de Banamex, así como para sus operaciones de redescuento, reflejada en un alto q. Esta mayor prima de liquidez se explica por la capacidad de mayor emisión (3 a I) y por el privilegio de la circulación de sus billetes a nivel nacional, todo lo cual le permite inyectar más liquidez al sistema para la financiación, el intercambio y las operaciones intrabancarias. Es por esto que el BLM tiene también un alto $q$ ya que también tiene los privilegios de circulación nacional y de emisión 3 a I, mientras que el BC alimenta las operaciones intrabancarias. Así, los parámetros asociados al modelo:

I3 Sólo para los bancos de Morelos y Michoacán resulta significativa una diferencia con respecto a la media nacional que los ubica en el rango de los q menores a la unidad. (ver anexo I Análisis de media I). 14 El cual puede escribirse como el sistema $q_{i}=q_{B X}+\mathrm{a}_{i}$ donde $i=B L M, B C$ y $B E$, donde $B E$ es la dummy para los otros bancos. (ver anexo I Análisis de media 3). 
(2) $q_{i, t}=1.3-0.12 B L M-0.11 B C-0.25 B E$

tienen una interpretación adicional como proxies de los costos de transacción que reducen la prima liquidez, es decir, la capacidad de inyectar liquidez al sistema por parte de los demás bancos.

\section{Gráfica 2}

Indicadores de confianza: Brecha depósitos a la vista vs a plazo $\left(B_{V P}\right)$ y brecha billetes vs depósitos $\left(B_{B D}\right)$ : 1905-1912

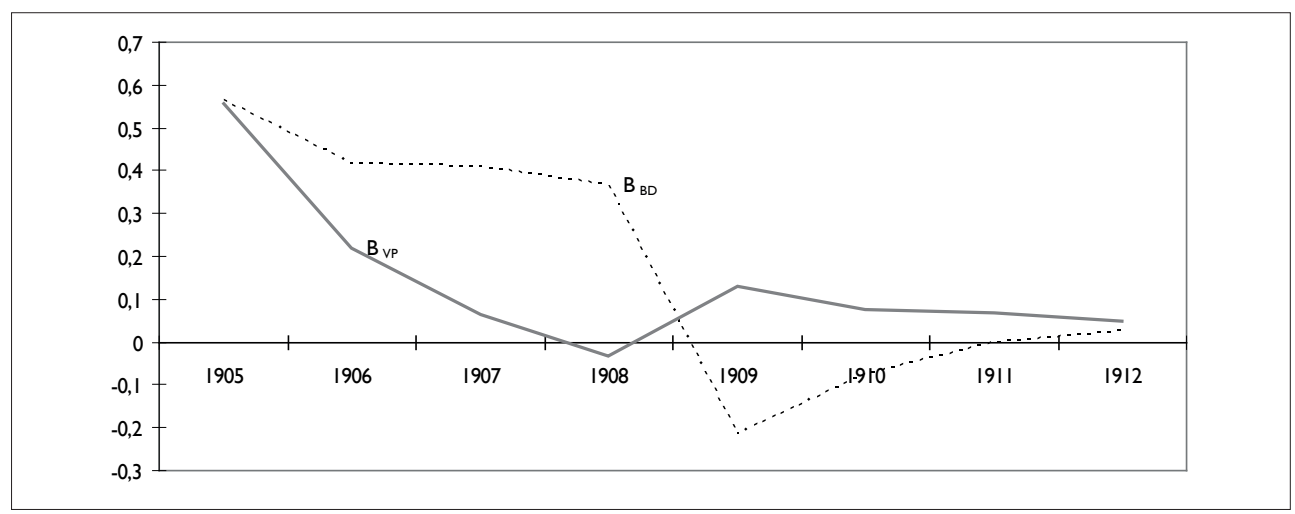

Fuente: Oñate (|99|). Cálculos del autor.

Una mirada macro hacia el estado de confianza puede darse evaluando las brechas entre depósitos a la vista vs a plazo $\left(B_{V P}\right)$ y billetes vs depósitos $\left(B_{B D}\right) \cdot{ }^{15} B_{V P}$ muestra como los depósitos a plazo fueron ganando peso entre 1905-1908, es decir, es evidencia de una mayor confianza en el sistema bancario, proceso que se trunca por la crisis de 1907 y que parece reiniciarse con la reforma a la ley de establecimientos de crédito de 1908, aunque ahora de una manera más lenta. $B_{B D}$ es el resultado conjunto de dos fenómenos por un lado la mayor aceptación de los billetes, lo cual permite ampliar la liquidez inyectada al sistema, así como de una postura más riesgosa por parte de los bancos, en particular de Banamex, la cual cambia con la crisis de 1907 (Maurer 2002). Con la crisis de 1907-1908 Limantour a evalúa el respaldo real de los medios de pago y propone la reforma a la ley general de instituciones de crédito promulgada en 1897, en esta propuesta de reforma "La idea fundamental, tratándose de bancos de emisión, consiste en procurar que el mecanismo de éstos sea tal, que el reembolso del billete de banco esté perfectamente garantizado en cualquier

I5 $B_{i, j}=\frac{V_{i} V_{j}}{V_{i} V_{j}}$, cuando $B_{i, j} \rightarrow 1$ mayor es el peso relativo del activo i; viceversa, cuando $B_{i, j} \rightarrow-1$ mayor es el peso relativo del activo j. 
momento..." Es claro que para la continuidad del Sistema de Pagos un requisito es que toda promesa de pago corresponda con la relación de activos y pasivos de los agentes que la componen, lo cual refuerza la confianza.

En cuanto a la banca refaccionaría e hipotecaria, la ley en sí misma no ofrece luces al respecto de los grados de liquidez de sus operaciones financieras, a saber, títulos de crédito y bonos. Sin embargo, podemos resaltar que su capacidad de inyectar liquidez a la economía fue bastante más restringida, como señala Limantour

\begin{abstract}
Salta a la vista, en primer término, la desproporción en el número de los bancos de emisión y de las demás instituciones de crédito fundadas a la sombra de la ley de 1897. La iniciativa particular y con ella los capitales nacionales y extranjeros, se han aplicado a multiplicar los documentos de crédito pagaderos a la vista y al portador como son los billetes de banco, de toda preferencia a los otros títulos de crédito que constituyen deudas a plazo y devengar réditos como los bonos de caja, los bonos hipotecarios, los certificados de los almacenes de depósitos, etc. Son muchos, quizá demasiado numerosos, los bancos de emisión que se han creado, e insuficientes los refaccionarios y los hipotecarios... (Limantour, 1908)
\end{abstract}

Con el avance en la construcción del sistema bancario y el rápido crecimiento del número de sociedades anónimas (Riguzzi, 2006), el mercado de valores empieza a tener presiones para su evolución. El mercado de valores (acciones y bonos) se encontraba evidentemente rezagado, Haber y Riguzzi argumentan que este rezago obedeció, en alto grado a la escasa (o nula) y esporádica información sobre los balances de las empresas (Riguzzi, 2006), de igual forma, afectó el hecho de que los bonos de deuda del gobierno incluyeran tasas con altísimas primas de riesgo y los privilegios de Banamex con relación a los demás títulos emitidos por el gobierno. Aunque en I887 se intentó iniciar la bolsa mercantil de México, ésta decayó rápidamente. Las actividades propias de una bolsa fueron suplidas por otro tipo de agentes, de los cuales podemos resaltar algunas casas comerciales, en la ciudad de México, que empezaron a realizar operaciones bursátiles en la calle a través de “corredores” (Jiménez, 2002). ${ }^{16}$ Posteriormente, en 1895 se creó la bolsa de México, con un fuerte apalancamiento en la minería, en " 5 meses logró el registro de 859 empresas mineras y 36 contratos, mismos que 10 años después empezaron a decaer arrastrando a la bolsa en su picada”. (Jiménez, 2002); para 1910 sus operaciones alcanzaron 10.2 millones de pesos, aunque cayeron vertiginosamente a 2.2 millones de pesos el año siguiente. Aunque los pequeños avances en el mercado de valores son contrastantes con

I6 De acuerdo al reglamento de corredores para la plaza México les autorizaba la inversión en títulos de crédito nacionales y extranjeros, documentos bancarios, acciones, operaciones en valores endosables y al portador, metales preciosos, amonedados o en pasta y también desempeñarse como peritos contables (Jiménez, 2002). 
los del sistema bancario, ${ }^{17}$ podemos avizorar efectos importantes en el sistema de pagos asociados a la reducción en los costos de transacción, en particular, en los costos de información, ya que el numero de empresas que presentaban balances como porcentaje de las sociedades cotizadas en el boletín de bolsa pasó de $50 \%$ en 1900 a $62 \%$ en 1910 (Riguzzi, 2006).

Este retrato a los principales componentes del sistema financiero, no es del todo completo cabría tomar en cuenta otros dos elementos: por un lado, un mercado informal de financiamiento conformado por redes tradicionales y que representaba la principal fuente de recursos para el crédito agrario; por el otro, casas bancarias no concesionadas, muchas veces de capital extranjero, que financiaban actividades de exportación y no estaban sujetas a la normatividad de la banca concesionada. Esto es importante porque después de la revolución, y en contraste con la lentitud con que se recompone el sistema crediticio, este fragmento del sector financiero ofrecerá un pequeño respiro a las actividades económicas. Estos dos elementos constituyen puntos para futura investigación.

\section{Moneda exógena y sistemas de pagos paralelos: | 9 |3-19|6}

Lo dicho hasta aquí, mantiene un punto al margen, a saber, la relación existente entre la elite política y la elite financiera. ${ }^{18}$ Entender la estabilidad de esta relación nos permite evaluar las estrategias de que disponían los actores en el Sistema de Pagos y las que tendrían los "nuevos" actores en el periodo revolucionario. Por está vía, podemos avanzar en la línea de Maurer (2002) y Haber et. al. (2003) con relación a la idea de que el régimen Porfiriano estableció un tipo de integración política vertical que dio estabilidad a los agentes que participaron de los acuerdos con el gobierno. La estructura oligopólica de la banca porfiriana es reflejo de la serie de privilegios de que gozarán los bancos, en particular, Banamex y Londres y México. Maurer (2002) enfatiza que los bancos del Porfiriato contaban con una amplia red de respaldo en la elite política, esta fue una condición para la sobrevivencia de un banco.Así, los privilegios de los bancos estatales dependían de su relación con el gobierno

17 Este tímido avance es resaltado por Haber et. al. cuando reseñan "In short, brokers and financial system did not play a particularly role in the Porfirian financial system. As a practical matter, the banks were the financial system" (Haber et. al. 2003).

18 Como señala Anaya "La estrecha relación entre política y banca ilustrada por el amplio número de gobernadores y funcionarios en los consejos de administración de los bancos de emisión muestra otra característica del atraso del sistema bancario mexicano. En consecuencia el código que normaba el comportamiento de los agentes financieros no se antoja fácilmente separable del que regía a las decisiones del mundo de la política" (Anaya, 2002, 43). 
estatal y mantenían una amplia línea de autopréstamos, mientras que los bancos nacionales obtenían privilegios por que mantenían líneas de crédito abiertas al Ejecutivo de la Unión y contaban con una poderosa red política de respaldo. ${ }^{19}$

Así, "The deal was not stable because the federal government passed a law in 1897 (o reformada en 1908). Porfirian Mexico was not governed by the rule of law. Rather, the arrangement was stable because once in placed none of the actors had an incentive to violate it." (Maurer, 2002, 4I) Por un lado, la concentración bancaria implicó su limitada capacidad para inyectar liquidez a la economía en su conjunto, como una extensión propia de un oligopolio que limita cantidades y de la práctica de los autopréstamos. Por otro lado, dicha concentración se apalancó en el respaldo político estratégico, que dio a la banca una amplia incidencia sobre la política monetaria.

La estabilidad de este acuerdo entró en prueba en el periodo revolucionario. En los periodos más álgidos de la lucha armada, los bancos fueron vistos como una fuente de liquidez y blanco de préstamos forzosos; ${ }^{20}$ y en los periodos de menor intensidad armada, fueron el blanco del ataque "ideológico" contra los representantes de la elite Porfiriana -el elemento que garantizaba sus derechos de propiedad ahora se volvía en su contra-. Ahora bien los bancos, en menor o mayor grado, eran concientes de las necesidades de liquidez de las facciones en pugna, lo cual representaba la apertura de un espacio para la reacción estratégica de los bancos. Para profundizar en el espacio de la capacidad de respuesta de la banca, veamos antes la raíz de este ataque ideológico. Desde el inicio los gobiernos revolucionarios plantearon la necesidad de transformar la estructura del sistema, Carranza plantea la necesidad abolir "el derecho de emisión de billetes o papel moneda, por bancos particulares. La emisión de billetes debe ser privilegio exclusivo de la nación.Al triunfo de la revolución establecerá el Banco Único de Emisión..." (Manero, 1957, 20I), idea que permanece latente en el ideario de los primeros gobiernos posrevolucionarios, aunque las bases de este proyecto sólo tendrían una expresión formal con la creación del Banco de México en 1925 y la Ley monetaria de 1931. Contrasta la idea de crear un Banco único de emisión, que implica un amplio control sobre el sistema de pago y la ampliación de las herramientas de política, con la idea limitada del Porfiriato de mantener la facultad de acuñar moneda exclusivamente en manos del Ejecutivo de la Unión.

I9 Por ejemplo, la familia de Limantour tenía intereses en el BLM, lo cual permitió que este accedería a privilegios.

20 Ver Maurer (2002) para una cuantificación de estos “préstamos”. 
Por otro lado, en el periodo revolucionario encontramos una plétora de emisiones y la convulsión del sistema de pagos asociada a éstas. Las facciones revolucionarias intentaron edificar su propio sistema de pagos a través de la acuñación de monedas y la emisión de billetes, ${ }^{21}$ cuya aceptación fue fugaz y dependió de cuanto tiempo se tuviera el control militar de cierta zona. Carranza intentó reordenar el sistema de pagos, en medio del conflicto con ciertas facciones revolucionarias; en este contexto, el espacio de estrategias de Carranza era muy limitado, requería de liquidez y buscaba transformar la estructura institucional del Sistema de Pagos heredada del Porfiriato. Así que su estrategia inicial fue la creación de la Comisión Reguladora e Inspectora de Instituciones de Crédito, en 1915, “Considerando que el interés público no está suficientemente garantizado respecto a la circulación de los billetes de los bancos de emisión”. (Manero, 1957, 202). En perspectiva al establecimiento del Banco Único de Emisión, esta comisión tuvo al menos tres objetivos estratégicos: (i) reestablecer el respaldo de las emisiones de la banca, (ii) solventar los problemas de confianza -por ello se reiteraba que seguía un "enfoque legalista"y, (iii) evaluar el sistema bancario de emisión, o en otros términos medir los recursos disponibles en el sistema bancario y la estructura de éste.

Un primer elemento que marca la dinámica de la comisión es la diferenciación de la política carrancista frente a las políticas del antiguo régimen y a otras facciones revolucionarias, en particular zapatistas y villistas. Como se señaló, la banca fue presa de préstamos forzosos y "voluntarios" con el gobierno de Huerta, ello era conocido por la Comisión, la cual anuló dichas deudas como respaldo de las emisiones bancarias; de igual manera los bancos tenían en su haber billetes de las facciones revolucionarias, así que la comisión exigió la presentación de éstos para su destrucción. Dicho esto, veamos algunos de los elementos que caracterizaban el sistema bancario en este periodo, tomados de la evaluación de la Comisión. Adelantamos, que el balance que encuentra la Comisión Reguladora e Inspectora de Instituciones de Crédito, no es muy diferente de los aspectos antes reseñados. Resalta la desaparición del Banco Central como agente de contrapeso a los Bancos Nacional y Londres y México, lo cual permitió la consolidación de éstos como bancos líderes, evidencia de ello es que para 1915 concentraban $67.57 \%$ del mercado de depósitos y billetes emitidos y $72.04 \%$ de existencias bancarias de metálico (Anexo II).

El índice de concentración industrial de $\mathrm{HH}$ ajustado en el mercado de depósitos y billetes alcanza 0.21 , muestra de la alta concentración en el sistema bancario, mientras que el mismo índice en términos de la existencia metálica alcanza 0.23 ; este último índice debe leerse con precaución, ya que no mide exactamente la con-

2I Los cuales revisten la simbología de la moneda con elementos propios de los mitos fundacionales de la revolución y de la cultura mexicana, acuñaciones que buscan y requieren enraizar la confianza en estos medios circulantes. 
centración industrial sino que más bien refleja el grado de cobertura frente al riesgo de las instituciones bancarias, es por esta razón que es mayor al índice anterior. Los bancos ajustados a ley mantienen en promedio $38.4 \%$ de la relación reservas y depósitos y billetes, ${ }^{22}$ con una participación en las reservas de $83.31 \%$; mientras que los bancos por fuera de la ley, mantienen en promedio $23.31 \%$ de la relación reservas y depósitos y billetes, con una participación en las reservas de $16.69 \%$ Esto muestra que los bancos que se mantuvieron dentro de la ley mantenían un mayor nivel de cobertura frente al riesgo que aquellos que fueron declarados en caducidad por la Comisión Reguladora. Esta última es la acción seguida por el gobierno con base en el balance realizado por la comisión, aunque en estricta atención a los datos, la declaración de caducidad tiene efectos menores" sobre el sistema de pagos, $y$ en efecto, puso en cintura al sistema bancario, si bien declaró en caducidad alrededor de $60 \%$ de las entidades de emisión, éstas sólo representaban $24.81 \%$ de los depósitos y efectivo y $16.69 \%$ de las existencia metálicas. Esta acción es el resultado del comportamiento político estratégico del gobierno y de la banca, el gobierno intentó mostrar su compromiso con las reglas establecidas, aunque ello implicó la aceptación de reglas porfirianas, mientras que la banca hizo válidos algunos de los acuerdos establecidos con el régimen Porfiriano.

La declaración de caducidad fue seguida por una segunda acción de mayor envergadura por parte del gobierno carrancista: la incautación bancaria de 1916, la cual tuvo dos efectos inmediatos: en primer lugar permitió el control de cerca de $\$ 77972$ I 34.4I en existencias metálicas y \$224994 2I4.75 de depósitos y billetes emitidos; con ello, obtuvo los fondos que le dieron respaldo a la emisión de los infalsificables. ${ }^{23}$ En segundo lugar, la incautación contribuyó a la creciente consolidación

22 Los Bancos Nacional, Londres y México y Nuevo León operan bajo concesión especial que les permitía emitir en proporción 3 a I de sus "existencia(s) en caja en dinero efectivo o en barras de oro - plata” Art 16 Ley 1897. El planteamiento de Limantour: "la acumulación de billetes de los distintos bancos en poder de otras instituciones del mismo género, práctica que aumenta ficticiamente la circulación" (Limantour 1908) indica que el dinero en efectivo se refiere a oro o plata acuñados así como a billetes de otros bancos. Aunque esta interpretación no sería la que tomaría la comisión reguladora de Carranza, ya que según la ley las monedas de oro y plata son las que cuentan con poder liberatorio y los billetes son de aceptación voluntaria.

23 "La medida no fue exitosa y la inflación pronto se transformó en hiperinflación. Sin embargo, tras este fracaso, el gobierno reconoció la nueva realidad y resolvió desmonetizar los infalsificables (que no valían en el mercado más que una fracción de su valor nominal). Por otra parte, era tal la desvalorización de los billetes en circulación que la población ya se negó a aceptar pagos en papel y comenzó a reclamar pagos en metálico. De allí que en relativamente poco tiempo, el papel fue desapareciendo de la circulación, siendo reemplazado por metálico en plata y oro que había estado atesorado, sobre todo por los sectores medios y altos,..., Esta tendencia que se hizo notar con fuerza a fines de 1916 , fue reforzada por el decreto del 29 de marzo, el cual estableció que se aceptarían infalsificables para el pago de impuestos a un valor superior al de mercado. El resultado fue que las empresas adquirieron una gran cantidad de este papel moneda para cubrir así sus obligaciones fiscales y para octubre de 1917, 
de las Casas Bancarias, proceso que venía evidenciándose desde 1913, supliendo las funciones de un sistema bancario “amedrentado", así como algunas del mercado de valores desarticulado. Cabe señalar que estas Casas operaban, principalmente, como matrices de Bancos extranjeros. No tenían concesión y por esto no eran considerados como bancos, tampoco se les hizo requerimientos especiales y su control era, básicamente, inexistente. ${ }^{24}$

Como un hecho sintomático de los intentos por tomar las riendas de la economía por parte de los gobiernos posrevolucionarios, la incautación tampoco fue una política estable, toda vez que ésta se revirtió para 1921, en medio de presiones externas y de la crisis financiera que atacaría en esta ocasión a las Casas Bancarias.

La crisis de 1921-1922, fue un escenario adverso para los empresarios, ${ }^{25}$ fenómeno que se acompañó y se reforzó por las expectativas pesimistas "de los poseedores pasivos de títulos y activos", generados por la inestabilidad política, los rezagos de los efectos causados por la inoperancia del ferrocarril, $y$, la alta volatilidad de los precios de la plata. ${ }^{26}$ Por otra parte, el gobierno de Obregón conservó una postura más pasiva en lo tocante al sistema de pagos, la cual en un principio se limitó a algunos acuerdos con las casas bancarias. ${ }^{27}$ Este panorama apuntaló la necesidad de edificar las bases de un sistema de pagos estable, tal y como se venía gestando desde el gobierno de Carranza, en el cual se proyectó insistentemente constituir las bases de un sistema de pagos moderno, cuya consolidación tendría que esperar hasta 1932.

\section{A modo de conclusión: \\ La propuesta de un nuevo sistema de pagos I917-1925}

En la búsqueda de elementos que permitan responder a la pregunta ien el tránsito del Porfiriato a los primeros gobiernos revolucionarios se logra establecer un arreglo institucional, en cuanto a una unidad de cuenta como forma monetaria capaz de desempeñar funciones de medio de pago y reserva de valor?, encontramos evidencia de un relativo avance en los elementos que componen el Sistema de Pagos. En el campo institucional, encontramos cambios en las leyes que reglamentaron y es-

la administración carrancista había logrado recoger más de 350 millones de pesos en infalsificables, lo cual alivió enormemente el problema monetario" (Marichal, 2004, 43).

24 A este repacto ver Anaya Merchant (2002) y Código de comercio 1884.

25 Ver Anaya Merchant (2002).

26 Ver Kemmerer 1917.

27 Acuerdos, en particular, con la Compañía Bancaria París México.Aunque estos acuerdos tardíos no surtirían el efecto deseado de detener ni desacelerar la corrida bancaria que avanzada en los primeros días de 1921, y que afectó con particular intensidad a dicha Compañía. (Anaya, 2002, 104 y ss.). 
tabilizaron en su momento el Sistema de Pagos.Vimos como la implementación de la Ley Monetaria de 1905, permite considerar las características del patrón oro y al papel de la plata como medio de pago. Se observó como la Ley de Establecimientos de Crédito de 1897 y su reforma en 1908, modificaron el ámbito institucional de operación de los agentes que interactuaban en el proceso de inyección de liquidez al sistema.

Aunque se evidenció relativa consolidación del Sistema de Pagos, ésta descansó sobre bases institucionales que limitaron su capacidad de expansión. La implementación del patrón no redujo la vulnerabilidad del Sistema frente a la alta volatilidad de los precios de la plata, lo cual fue particularmente importante en el periodo de 1916-1920. La alta concentración en el sistema bancario y los privilegios que ella implicó, lastraron las posibilidades de expansión del crédito doméstico. ${ }^{28}$ Y, finalmente, el estancamiento del mercado de valores y su crisis en los inicios de la revolución. Todos ellos, elementos de la matriz institucional que heredaron los gobiernos posrevolucionarios.

El limitado espacio estratégico de los gobiernos revolucionarios es también el resultado de las características reseñadas. El empeño puesto desde 1915 en la propuesta de consolidación de un Banco Único de Emisión, choca con la inestabilidad política que caracterizó al México de la época y con la profunda red de reglas y organizaciones fortalecidas en el Porfiriato. Así, la propuesta que emerge de este escenario como reflejo de esta situación, puede presentarse en dos grandes líneas: una primera, en perspectiva a las estrategias de los gobiernos posrevolucionarios con relación al sistema de pagos, y, la segunda, en consonancia con la propuesta de Kemmerer con relación al sistema monetario, lo cual nos permite tener una visión de conjunto de las opciones de propuesta del Sistema de Pagos mexicano.

Fueron muy variadas las estrategias que usaron los distintos gobiernos posrevolucionarios para tratar de avanzar en la consolidación del sistema bancario, sin embargo, el efecto de las acciones desarrolladas en este periodo, normalmente fue poco duradero. Podemos destacar la emisión de los "infalsificables" como un intento por instaurar el dinero fiduciario en México, pero esta política en los hechos fue el reflejo de la débil confianza que los agentes depositaban en formas monetarias diferentes a la plata $\circ$ al oro. Por esta razón, la propuesta resultante de creación del Banco de México tiene que seguir otro tipo de estrategias que se concretaron en una amplia publicidad de las propuestas del gobierno a través de la prensa y la

28 Como un hecho sintomático de estos privilegios y las restricciones que imponían sobre el sistema crediticio resalta la creación de la Caja de Préstamos para Obras de Irrigación y Fomento de la Agricultura, la cual inyectó liquidez al Sistema de Pagos por la vía de los privilegios. Los recursos que en principio debieron ser inyectados como resultado de una política de crédito productivo, fueron usados para “sanar" la cartera de los Bancos más privilegiados (Maurer 2002, 66-68). 
contratación de asesores externos para este proceso. Por otro lado, la propuesta también tenía que combatir la alta concentración del sistema bancario mexicano, por lo cual optó por un Banco Central con una alta presencia a escala estatal, éste actuaría como una suerte de banco privado que competiría con la banca privada. En el espectro institucional, era necesaria la reforma del artículo 20 de la Constitución de 1917,y elementos legales que le permitieran garantizar la aceptación de nuevas formas monetarias, como el que se consolida en una reforma posterior del Artículo I 23 de la Constitución que establece el pago de salarios con papel moneda.

Esta última preocupación, tiene que ver con el hecho reseñado por Kemmerer "It was the sudden and unexpected return from hoards into active circulation of an enormous volume of gold and silver coin, driving out of circulation practically all the paper money and placing the country squarely back upon the gold standard- and all within the surprisingly short period of a few days." (Kemmerer, 1940). Este fenómeno fue generado por la desconfianza en los "infalsificables" y demuestra la sólida confianza en la plata y el oro. Es por esto que para Kemmerer resultaba necesario reestablecer la continuidad del patrón oro, en la medida en que era necesario que la acuñación se ajustara automáticamente al volumen de la actividad económica. Por esto planteó que las casas de moneda debían operar como lo hicieron en el Porfiriato, acuñando moneda cuando los agentes lo requirieran. La creación y la conservación de la confianza es un elemento vital de la propuesta y ella requería contrarrestar los efectos de la volatilidad de los precios de la plata y el oro. 


\section{ANEXO I}

Análisis de media I

\begin{tabular}{|c|c|c|c|c|c|c|}
\hline Source & SS & df & Ms & & \multicolumn{2}{|c|}{ Number of obs= } \\
\hline Model Residual & $\begin{array}{l}\text { I.25202229 } \\
\text { I.0398000। }\end{array}$ & $\begin{array}{c}24 \\
200\end{array}$ & $\begin{array}{c}.052167595 \\
.005199\end{array}$ & & \multirow{2}{*}{\multicolumn{2}{|c|}{$\begin{array}{c}F(24,200)= \\
\text { Prob }>F= \\
\text { R-squared = } \\
\text { Adj. } \text { R-squared = }\end{array}$}} \\
\hline Total & 2.2918223 & 224 & 0.01023135 & & & \\
\hline$q$ & Coef. & Std. Err. & $\mathrm{t}$ & $P>|t|$ & \multicolumn{2}{|c|}{ [95\% Conf. Interval] } \\
\hline BLM & -.1222222 & .0339902 & -3.60 & 0.000 & -1892473 & $-.055|97|$ \\
\hline$B C$ & -.1166666 & .0339902 & -3.43 & 0.001 & -.1836918 & -.0496415 \\
\hline Hipotecario & -.1955556 & .0339902 & -5.75 & 0.000 & -.2625807 & .1285304 \\
\hline Aguascalientes & -.2888889 & .0339902 & -8.50 & 0.000 & $-.3559 \mid 4$ & .2218637 \\
\hline Coahuila & -.2722222 & .0339902 & -8.01 & 0.000 & -.3392474 & .2051971 \\
\hline Durango & -.2544445 & .0339902 & -7.49 & 0.000 & -.3214696 & .1874193 \\
\hline Edo. México & -.2655556 & .0339902 & -7.81 & 0.000 & -.3325807 & .1985304 \\
\hline Guanajuato & -.2277778 & .0339902 & -6.70 & 0.000 & -.2948029 & .1607526 \\
\hline Hidalgo & -.2355555 & .0339902 & -6.93 & 0.000 & -.3025807 & .1685304 \\
\hline Jalisco & -.2533333 & .0339902 & -7.45 & 0.000 & -.3203585 & -.1863082 \\
\hline Minero & -.2933333 & .0339902 & -8.63 & 0.000 & -.3603585 & -.2263082 \\
\hline Monterrey & -.2377778 & .0339902 & -7.00 & 0.000 & -.3048029 & -.1707526 \\
\hline Veracruz & -.2133333 & .0339902 & -6.28 & 0.000 & -.2803585 & -.1707526 \\
\hline Morelos & -.3122222 & .0339902 & -9.19 & 0.000 & -.3792473 & $-245|97|$ \\
\hline Nuevo León & -.15111111 & .0339902 & -4.45 & 0.000 & -.2181363 & -.084086 \\
\hline Oriental & -.2788889 & .0339902 & -8.24 & 0.000 & $-.3459 \mid 4$ & -.2118638 \\
\hline Occidental & -.1666667 & .0339902 & -4.90 & 0.000 & -.2336918 & -.0996415 \\
\hline Querétaro & -.2944444 & .0339902 & -8.66 & 0.000 & -.3614696 & -.2274193 \\
\hline Sonora & -.2177778 & .0339902 & $-6.4 I$ & 0.000 & -.2848029 & -.2274193 \\
\hline SLP & -.24 & .0339902 & -7.06 & 0.000 & $-.307025 \mathrm{I}$ & -.1729749 \\
\hline Tabasco & -.2388889 & .0339902 & -7.03 & 0.000 & -.305914 & -.1718637 \\
\hline Tamaulipas & -.2988889 & .0339902 & -8.79 & 0.000 & -.365914 & -.2318637 \\
\hline Zacatecas & -.2255555 & .0339902 & -6.64 & 0.000 & -.2925807 & -.1585304 \\
\hline Michoacán & -.3711111 & .0339902 & -10.92 & 0.000 & $-.438 \mid 363$ & -.304086 \\
\hline _cons & 1.3 & .0339902 & 51.09 & 0.000 & 1.252606 & I.347394 \\
\hline
\end{tabular}


Análisis de media 2

\begin{tabular}{|c|c|c|c|c|c|c|}
\hline Source & SS & $d f$ & Ms & & \multirow{3}{*}{\multicolumn{2}{|c|}{$\begin{array}{c}\text { Number of obs }=225 \\
F(24,200)=10.03 \\
\text { Prob }>F=0.0000 \\
\text { R-squared }=0.5463 \\
\text { Adj. R-squared }=0.4919 \\
\text { Root MSE }\end{array}$}} \\
\hline Model Residual & $\begin{array}{l}\mathrm{I} .25202229 \\
\mathrm{I} .0398000 \mathrm{I}\end{array}$ & $\begin{array}{r}24 \\
200\end{array}$ & $\begin{array}{r}.052167595 \\
.005199\end{array}$ & & & \\
\hline Total & 2.2918223 & 224 & 0.01023135 & & & \\
\hline $\mathrm{q}$ & Coef. & Std. Err. & $\mathrm{t}$ & $P>|t|$ & \multicolumn{2}{|c|}{ [95\% Conf. Interval] } \\
\hline$B L M \subset$ & .1086667 & .0235491 & 4.61 & 0.000 & .0622303 & $.|55| 03 \mid$ \\
\hline $\mathrm{BC} \subset$ & .1142222 & .0235491 & 4.85 & 0.000 & .0677859 & 0.1606586 \\
\hline Hipotecarioc & .0353333 & .0235491 & 1.50 & 0.135 & -.011103 & .0817697 \\
\hline Aguascalientes $\subset$ & -.58 & .0235491 & -2.46 & 0.015 & -.1044364 & -.0115636 \\
\hline Coahuila $\subset$ & $-.04 \mid 334$ & ا & -1.76 & 0.081 & -.0877697 & .005103 \\
\hline Durangoc & -.235556 & .0235491 & -1.00 & 0.318 & -.0699919 & .0228808 \\
\hline Edo. México & -.346667 & .023549 & -1.47 & 0.143 & $-.08|103|$ & .0117697 \\
\hline Guanajuatoc & .0031111 & .0235491 & 0.13 & 0.895 & -.0433253 & .0495475 \\
\hline Hidalgo $\subset$ & -.0046666 & .0235491 & -0.20 & 0.843 & -.051103 & .0417697 \\
\hline Jalisco $\subset$ & -.0224444 & .0235491 & -0.95 & 0.342 & -.0688808 & .0239919 \\
\hline Minero $\subset$ & -.0624444 & .0235491 & -2.65 & 0.009 & -.1088808 & -.0160081 \\
\hline Monterrey $\subset$ & -.0068889 & .023549I & -0.29 & 0.770 & -.0533253 & .0395475 \\
\hline Veracruz $\subset$ & .0175555 & .0235491 & 0.75 & 0.457 & -.0288808 & .0639919 \\
\hline Morelos $\subset$ & -.0813333 & .0235491 & -3.45 & 0.001 & -.1277697 & -.0348969 \\
\hline Nuevo Leónc & .0797778 & .0235491 & 3.39 & 0.001 & .0333414 & .1262141 \\
\hline Orientalс & -.048 & .0235491 & -2.04 & 0.043 & -.0944364 & -.0015636 \\
\hline Occidental $\subset$ & .0642222 & .0235491 & -2.04 & 0.043 & -.0944364 & -.0015636 \\
\hline Querétaroc & -.0635556 & .0235491 & -2.70 & 0.008 & -.1099919 & -.0171192 \\
\hline Sonora & .0131111 & .0235491 & 0.56 & 0.578 & -.0333253 & .0595475 \\
\hline$S L P \subset$ & -.0091111 & .0235491 & -0.39 & 0.699 & -.0555475 & .0373253 \\
\hline Tabasco $\subset$ & -.008 & .0235491 & -0.34 & 0.734 & -.0544364 & -0384364 \\
\hline Tamaulipas $\subset$ & -.068 & .0235491 & -2.89 & 0.004 & -.1144364 & -.0215636 \\
\hline Zacatecas $\subset$ & .0053333 & .0235491 & 0.23 & 0.821 & -.041103 & .0517697 \\
\hline Michoacánc & -.1402222 & .0235491 & -5.95 & 0.000 & -.1866586 & -.937859 \\
\hline _cons & 1.069111 & .0048069 & $222.4 \mathrm{I}$ & 0.000 & 1.059632 & 1.07859 \\
\hline
\end{tabular}


Análisis de media 3

\begin{tabular}{|c|c|c|c|c|c|c|}
\hline Source & SS & $d f$ & MS & & \multirow{4}{*}{\multicolumn{2}{|c|}{$\begin{array}{c}\text { Number of obs }=225 \\
F(3,22 I)=38.58 \\
\text { Prob }>F=0.0000 \\
\text { R-squared }=0.3437 \\
\text { Adj. } \text { R-squared }=0.3348 \\
\text { Root } M S E=0.825\end{array}$}} \\
\hline Model & .787721302 & 3 & .262573767 & & & \\
\hline Residual & 1.50410099 & 221 & .006805887 & & & \\
\hline Total & 2.2918223 & 224 & .01023135 & & & \\
\hline$q$ & Coef. & Std. Err. & $\mathrm{t}$ & $P>|t|$ & \multicolumn{2}{|c|}{ [95\% Conf. interval] } \\
\hline BLM & -.1222222 & .0388898 & -3.14 & 0.002 & -.1988646 & -.0455798 \\
\hline$B C$ & -.1166666 & .0388898 & -3.00 & 0.003 & -.193309 & -.0400243 \\
\hline BE & -.2515152 & .0281173 & -8.95 & 0.000 & -.3069275 & -.1961028 \\
\hline _cons & 1.3 & .0274993 & 47.27 & 0.000 & 1.245806 & 1.3541194 \\
\hline
\end{tabular}




\section{ANEXO II}

Situación de liquidez de los bancos de emisión: 1915 (Porcentajes)

\begin{tabular}{|c|c|c|c|}
\hline Bancos ajustados a ley & $\frac{R}{E+D *}$ & $\begin{array}{c}\text { Participación en el mercado } \\
\text { de depósitos y emisión de } \\
\text { billetes }\end{array}$ & $\begin{array}{l}\text { Participaciónen } \\
\text { Existencias } \\
\text { Metálicas }\end{array}$ \\
\hline Banco Nacional & 37.64 & 40.18 & 43.64 \\
\hline Banco de Londres & 35.93 & 27.39 & 28.40 \\
\hline Banco de Zacatecas & 50.21 & 0.68 & 0.99 \\
\hline Banco Estado de México & 50.46 & 1.39 & 2.02 \\
\hline Banco de Nuevo León & 39.39 & 0.99 & 1.12 \\
\hline Banco de Tabasco & 50.90 & 0.49 & 0.72 \\
\hline Banco de Veracruz & 56.39 & 2.05 & 3.33 \\
\hline Banco de Sonora & 52.98 & 1.03 & 1.58 \\
\hline Banco Occidental de México & 52.73 & 0.99 & 1.51 \\
\hline Subtotal Ajustados a la ley & 38.40 & 75.19 & 83.31 \\
\hline \multicolumn{4}{|l|}{ Bancos en caducidad } \\
\hline Banco Peninsular & 24.57 & 2.47 & 1.75 \\
\hline Banco de Hidalgo & 33.57 & 0.73 & $0.7 \mathrm{I}$ \\
\hline Banco de Guerrero & 21.05 & 0.30 & 0.18 \\
\hline Banco de Querétaro & 24.51 & 0.93 & 0.66 \\
\hline Banco de San Luis Potosí & 33.32 & 1.30 & 1.25 \\
\hline Banco de Coahuila & 23.72 & 2.04 & 1.40 \\
\hline Banco Oriental & 22.28 & 12.44 & 8.00 \\
\hline Banco de Jalisco & 25.94 & 1.66 & 1.24 \\
\hline Banco de Tamaulipas & 25.03 & 2.02 & 1.46 \\
\hline Banco Mercantil de Monterrey & 0.99 & 0.90 & 0.03 \\
\hline Subtotal en caducidad & 23.31 & 24.81 & 16.69 \\
\hline Total & 34.66 & 100.00 & 100.00 \\
\hline
\end{tabular}

Fuente: Manero (1957). Cálculos del autor. Los bancos de Aguascalientes, Guanajuato, Morelos, Durango y Chihuahua no presentaron información y fueron considerados en "rebeldía".

*Existencias metálicas ( $R=$ reservas), Depósitos y billetes en circulación $E+D$ 


\section{Bibliografía}

Anaya, Luis (2002), Colapso y reforma: La integración del sistema bancario en el México revolucionario 19/3-1932. Universidad de Zacatecas y Editorial Porrua.

Borja, Francisco (1990), La reforma Monetaria de 1905. Escuela Libre de Derecho y editorial Porrua.

Graziani, Augusto (1990), La teoría del circuito monetario. En coyuntura colombiana 55, vol. 14 No.3, septiembre de 1997.

Haber, Maurer y Razo (2003), The Politics of Property Rights: Political Instability, Credible Commitments, and Economic Growth in Mexico, 1876-1929. Cambridge University Press.

Jiménez, Sergio Raúl (2000), La Bolsa de Valores en México:Antecedentes y Situación Actual. http://www.profmex.org/mexicoandtheworld/volume5/I winter00/labolsa_devalores. html Consultado 27 de mayo de 2010.

Kemmerer, Edwin. (1917), Sistema Monetario de México Reformas propuestas por el señor E.W. Kemmerer. Palacio Nacional 1917.

(1940), Inflation and Revolution: Mexico's Experiences of 19/2-1917, Princeton University Press.

(1959), Oro y patrón oro, Editorial Sudamericana Buenos Aires.

Keynes, John. (1937), El financiamiento de Mr. Keynes. En coyuntura colombiana 55, vol. I4 No.3, septiembre de 1997.

Limantour, José (1908), Exposición de motivos presentada por José Y. Limantour, Secretario de Hacienda. En Ludlow, Leonor y Marichal, Carlos (coordinadores) (1998).

Lorente, Luís (1991), Políticas monetarias y estancamiento económico. En Coyuntura Colombiana 55, vol. 14 No.3, septiembre de 1997.

Ludlow, Leonor y Marichal, Carlos (coordinadores) (1998) La Banca en México, I820-I920. Instituto Mora, El Colegio de Michoacán, El Colegio de México e Instituto de Investigaciones Históricas-UNAM.

Ludlow, Leonor y Salmeron, Alicia (1997), La emisión del papel moneda en México: Una larga negociación político-financiera, Secretaria de Hacienda y Crédito Público.

Manero, Antonio (1957), Iniciación de la reforma Bancaria, 1913. En Ludlow, Leonor y Marichal, Carlos (coordinadores) (1998).

Marichal, Carlos (2004), Debates sobre los orígenes de la banca central en México, El Colegio de México.

Maurer, Noel (2002), The Power and the Money: The Mexican Financial System, I876-1932, Stanford University Press.

Rachline, Francois (1982), La nature de la monnaie. Revue économique, Vol. 33, No. 3. (May, 1982), pp. 446-475. 
Riguzzi, Paolo (2006), Sistema legal y sociedades anónimas en México, I854-1917. El Colegio Mexiquense.

Romero, María Eugenia (compiladora) (2007), Actas de las juntas generales y documentos a ellas anexos. Reforma monetaria de 1905, UNAM.

Schell, William (1996), Money as a Commodity: Mexico's Conversion to the Gold standard. 1905, University of California Press. http://www.jstor.org/stable/I052078 Consultado el 20/01/2010.

Téllez Mario y López José (compiladores), La legislación Mexicana de Manuel Dublán y José María Lozano,disco compacto. El Colegio de México.

Wray, Randall (1993), The Origins of Money and the Development of the Modem Financial System, Working Paper No 86. Jerome Levy Economics Institute of Bard College and University of Denver

Zebadúa, Emilio (2004), Banqueros y revolucionarios: la soberanía financiera de México, 19/41929, El Colegio de México y Fondo de Cultura Económica. 